\title{
Rectosigmoid Cancer by AJCC v7 Stage
}

National Cancer Institute

\section{Source}

National Cancer Institute. Rectosigmoid Cancer by A/CC v7 Stage. NCI Thesaurus. Code C91226.

A term that refers to the staging of rectosigmoid carcinoma according to the American Joint Committee on Cancer, 7th edition. 